OPEN ACCESS

Edited by:

Chi-Kuang Wen,

Shanghai Institutes for Biological

Sciences (CAS), China

Reviewed by:

Lei Wang,

Institute of Botany (CAS), China

Mingyi Jiang,

Nanjing Agricultural University, China

${ }^{*}$ Correspondence:

Hongning Tong

tonghongning@caas.cn

Chengcai Chu

ccchu@genetics.ac.cn

tThese authors have contributed

equally to this work.

Specialty section: This article was submitted to

Plant Physiology,

a section of the journal

Frontiers in Plant Science

Received: 30 July 2017 Accepted: 15 September 2017

Published: 27 September 2017

Citation:

Xiao Y, Liu D, Zhang G, Tong $H$ and Chu C (2017) Brassinosteroids

Regulate OFP1, a DLT Interacting Protein, to Modulate Plant Architecture and Grain Morphology in Rice. Front. Plant Sci. 8:1698. doi: 10.3389/fpls.2017.01698

\section{Brassinosteroids Regulate OFP1, a DLT Interacting Protein, to Modulate Plant Architecture and Grain Morphology in Rice}

\author{
Yunhua Xiao ${ }^{1,2 t}$, Dapu Liu' ${ }^{1,2 t}$, Guoxia Zhang ${ }^{1,2}$, Hongning Tong ${ }^{3 *}$ and Chengcai Chu ${ }^{1,2 *}$ \\ 'State Key Laboratory of Plant Genomics and Center for Plant Gene Research, Institute of Genetics and Developmental \\ Biology, Chinese Academy of Sciences, Beijing, China, ${ }^{2}$ University of Chinese Academy of Sciences, Beijing, China, \\ ${ }^{3}$ National Key Facility for Crop Gene Resources and Genetic Improvement, Institute of Crop Sciences, Chinese Academy of \\ Agricultural Sciences, Beijing, China
}

Brassinosteroids (BRs) regulate important agronomic traits in rice, including plant height, leaf angle, and grain size. However, the underlying mechanisms remain not fully understood. We previously showed that GSK2, the central negative regulator of BR signaling, targets DLT, the GRAS family protein, to regulate BR responses. Here, we identified Ovate Family Protein 1 (OFP1) as a DLT interacting protein. OFP1 was ubiquitously expressed and the protein was localized in both cytoplasm and nucleus. Overexpression of OFP1 led to enlarged leaf angles, reduced plant height, and altered grain shape, largely resembled $D L T$ overexpression plants. Genetic analysis showed that the regulation of plant architecture by OFP1 depends on DLT function. In addition, we found OFP1 was greatly induced by BR treatment, and OsBZR1, the critical transcription factor of BR signaling, was physically associated with the OFP1 promoter. Moreover, we showed that gibberellin synthesis was greatly repressed in OFP1 overexpression plants, suggesting OFP1 participates in the inhibition of plant growth by high BR or elevated BR signaling. Furthermore, we revealed that OFP1 directly interacts with GSK2 kinase, and inhibition of the kinase activity significantly promotes OFP1 protein accumulation in plant. Taken together, we identified OFP1 as an additional regulator of BR responses and revealed how BRs promote OFP1 at both transcription and protein levels to modulate plant architecture and grain morphology in rice.

Keywords: DLT, grain shape, GSK2, leaf angle, OFP, plant height, rice

\section{INTRODUCTION}

Brassinosteroids (BRs) are a class of phytohormones playing important roles in regulating various aspects of plant growth and development. In the past decades, rapid progresses have been made regarding the hormone signaling in the model plant Arabidopsis. A nearly complete primary signaling pathway has been established from the receptor to the transcriptional factors involving multiple components including BRI1 receptor kinase, BAK1 co-receptor kinase, BKI1 receptor inhibitor kinase, BSK1 and CDG1 kinases, BSU1 phosphatase, BIN2 kinase, PP2A phosphatase, BZR1/BES1 transcriptional factors, and so on (Gruszka, 2013; Vriet et al., 2013). Among them, BIN2 is the central negative regulator which directly inhibits BZR1/BES1 activity (He et al., 2002; 
Yin et al., 2002). Recently, KIB1 was identified as a negative regulator of BR signaling responsible for BIN2 degradation, and multiple components including SINAT and DSK were identified as positive regulator of BR signaling participated in BZR1/BES1 degradation (Nolan et al., 2017; Yang and Wang, 2017; Yang et al., 2017; Zhu et al., 2017). In addition, a number of additional BIN2 targets as well as BES1 interacting proteins were reported, which are involved in diverse biological processes, further complemented the signaling network and greatly advanced our understanding of BR functional mechanisms (Hao et al., 2013; Youn and Kim, 2015).

In crop plant rice, BRs play critical roles in regulating plant height, leaf angle, and grain size. Because these characteristics are important agronomic traits that mostly considered in breeding activity, BRs are thought to have great potentials in agricultural improvement (Divi and Krishna, 2009). Several studies have tried to utilize BR-related plants or genes for crop engineering and obtained promising results. For example, a weak BRdeficient plant osdwarf4, or an OsBRI1-cossuppression plant could have greatly enhanced population yield when planting at high density due to their favorable erect leaves (Morinaka et al., 2006; Sakamoto et al., 2006). However, since rice has a distinct plant architecture from Arabidopsis, it remains largely unclear how BRs specifically regulate these traits in detail in rice. Consistently, although some counterparts of the BR primary signaling components including OsBRI1, GSK2 (OsBIN2), and OsBZR1 were identified and their function were verified or partially verified, several rice specific BR components were also reported, including DLT, LIC, SMOS1/RLA1, and OFP8 (Wang et al., 2008; Tong et al., 2009; Tong and Chu, 2012; Zhang et al., 2012; Yang et al., 2016; Hirano et al., 2017; Qiao et al., 2017).

OFPs, for short of Ovate Family Proteins, are plant specific transcription factors containing a common domain named OVATE or DUF623 (domain of unknown function) (Wang et al., 2007, 2011). In rice, it should be noted that there were two genome-wide characterization studies of OFPs, which adopted different numbering systems of the family members (Liu et al., 2014; Yu et al., 2015). Founding member of the family, OVATE, was cloned in tomato, which plays crucial roles in regulating fruit shape (Liu et al., 2002). A naturally existed nonsense mutation in the gene in tomato varieties leads to the shift of roundshape fruit to pear-like shape. Surprisingly, consequent studies in Arabidopsis revealed that loss-of-function plants of many single or multiple OFP members are phenotype-silent (Wang et al., 2007, 2011; Schmitz et al., 2015). In contrast, overexpression of some OFP members produced evident morphology changes, suggesting the high redundancy among the family members. Overexpression of AtOFP1 in Arabidopsis produced dwarf statue due to the repression of gibberellin (GA) synthesis (Wang et al., 2007). AtOFP1 directly targets GA20ox-1, a GA biosynthetic gene, to inhibit its expression. Similarly, overexpression of OsOFP2 in rice also inhibited GA synthesis and decreased plant height likely by regulating GA20ox-7 (Schmitz et al., 2015). In addition, OFPs tend to generally interact with BELL- and KNOX-like proteins to regulate plant growth and development (Hackbusch et al., 2005; Schmitz et al., 2015). Recently, OFP8, corresponding to OFP30 in Liu et al. (2014), was shown to be involved in BR responses (Yang et al., 2016). Enhanced expression of the gene caused by T-DNA insertion containing 35S enhancer produced obviously enlarged leaf angles and increased $\mathrm{BR}$ responses. The protein localization was modulated by GSK2 phosphorylation, similar as the regulation of BZR1/BES1 by BIN2 (Yang et al., 2016).

More and more studies suggested that functions of BRs could be variable depending on species, tissues, hormone levels, developmental stages, and cell types (Singh and SavaldiGoldstein, 2015; Tong and Chu, 2016; Unterholzner et al., 2016). For example, BRs were reported to repress stomata development in cotyledons but promote the process in hypocotyls in Arabidopsis (Gudesblat et al., 2012; Kim et al., 2012). As growth-promoting hormones, BRs promote GA accumulation by inducing the expression of GA biosynthetic genes to stimulate cell elongation under physiological conditions (Tong et al., 2014; Unterholzner et al., 2015), however, sufficient high BR level or signaling will inhibit cell elongation and plant growth partially by repressing GA synthesis in rice (Tong et al., 2014). The mechanisms underlying this kind of functional specificities of BRs remain largely unclear.

In this study, we identified OFP1 as a DLT interacting protein by yeast two-hybrid screening, and found that OFP1 can also interact with GSK2 kinase. We showed that OFP1 was regulated by BRs at both transcription and protein levels, and the regulation was closely linked to the core BR signaling components involving GSK2, OsBZR1, and DLT. Our results revealed the positive roles of OFP1 in regulating $\mathrm{BR}$ responses to modulate plant architecture and grain morphology.

\section{MATERIALS AND METHODS}

\section{Plant Materials, Growth Conditions, and Chemical Treatment}

Japonica cultivars Zhonghua 11 (ZH11) or Dongjin (DJ) was used as the wild type (WT) for the transgenic analyses. Plants were grown on soil in field or in greenhouse or on $0.5 \mathrm{x}$ Murashige and Skoog $(1 / 2 \mathrm{MS})$ medium in a conditioned growth chamber at $30^{\circ} \mathrm{C}$ for $10 \mathrm{~h}$ (day) and $24^{\circ} \mathrm{C}$ for $14 \mathrm{~h}$ (night). For BR induction experiments, young seedlings were transferred to $1 / 2 \mathrm{MS}$ medium supplemented with various concentrations of brassinolide (BL, one of the active BRs) for $48 \mathrm{~h}$. For lamina inclination assay, ethanol $(1 \mu \mathrm{L})$ containing various amount of $\mathrm{BL}$ was spotted onto the top of lamina after 2-day germination and 3-day growth at $30^{\circ} \mathrm{C}$ (Tong and $\mathrm{Chu}, 2017$ ). Images were taken after 3-day incubation, and the angles of lamina joint bending were measured using IMAGEJ software ${ }^{1}$. For bikinin treatment, 1 -week-old seedlings were grown in $1 / 2 \mathrm{MS}$ supplemented with or without $30 \mu \mathrm{M}$ chemical for 3 days before sampling.

\section{Vector Constructions and Transgenic Analysis}

The DNA sequence containing the promoter and coding sequence of OFP1 was cloned into PCAMBIA2300 vector for

\footnotetext{
${ }^{1}$ http://rsb.info.nih.gov/ij
} 
OFP1 overexpression (OFP1-OE or 1o). A pCAMBIA230035S-GFP vector was used for OFP1-GFP construction. A pCAMBIA1300-35S-Flag vector was used for OFP1-Flag overexpression (OFP1-Flag-OE or $1 F o$ ). OFP1 promoter (2-kb) was introduced into $p C A M B I A 2391 Z$ vector for OFP1p:GUS construction. CRISPR/Cas9 knock-out vectors were constructed following previous reports (Ma and Liu, 2016). Primer sequences used for vector constructions and additional plasmid information are listed in the Supplementary Table 1. Sequences were introduced into vectors by either in-fusion cloning strategy (Clontech) or traditional cut-ligation cloning method. These constructs were used to transform rice plant or to infiltrate tobacco leaf epidermis cells by Agrobacterium-mediated method (Sparkes et al., 2006).

\section{Protein: Protein Interaction Analyses}

Yeast two-hybrid tests were performed following standard procedures descried by manufacturer (Clontech). BiFC (bimolecular fluorescence complementation) analyses were performed following the method described previously (Waadt et al., 2008). Rice protoplast preparation and plasmid transfection were performed according to the previous method (Bart et al., 2006). Fluorescence was observed on a confocal fluorescence microscope (Leica TCS SP6). Split-luciferase complementation assays were performed in tobacco leaves as described (Chen et al., 2008). Chemiluminiscence was photographed using a imaging system equipped with a cold CCD (NightOWL II LB983 with indigo software). See Supplementary Table 1 for the information of the primer sequences and plasmids used for the vector constructions prepared for the above analyses.

\section{Gene Expression and Promoter Activity Analysis}

RNA was isolated using Trizol reagent (Invitrogen) according to manufacturer's instruction. The first-strand cDNA was synthesized using a Revertase Transcription kit (Toyoba). Quantitative RT-PCR (qRT-PCR) was performed on a realtime PCR detection system following manufacturer's instructions (Bio-Rad CFX96). Rice Ubiquitin2 gene (UBQ) was used as internal control for all analyses. Primer sequences are listed in the Supplementary Table 2. GUS staining was performed according to previous description (Tong et al., 2009).

\section{Immunoblotting and ChIP-qPCR Analysis}

Commercialized anti-Flag (Sigma) and anti-ACTIN (Abmart) antibodies were used for immunoblotting analyses. Commercialized anti-OsBZR1 antibody (BPI) was used for ChIP (chromatin immunoprecipitation) analysis. ChIP-qPCR (ChIP followed by quantitative PCR analysis) was performed according to previous description (Tong et al., 2014). Primer sequences are listed in the Supplementary Table 2.

\section{GA Measurement}

About $4 \mathrm{~g}$ of shoots was harvested from the rice seedlings for GA measurements. Quantification of endogenous GAs was performed as described (Chen et al., 2012).

\section{RESULTS}

\section{OFP1 Interacts with DLT in Yeast}

We previously showed that GSK2 kinase, the central negative regulator of BR signaling, targets DLT, the GRAS family transcription factor, to regulate downstream BR responses (Tong et al., 2012). To further explore the functional mechanism of the proteins, we performed extensive yeast two-hybrid screenings of the interacting proteins using DLT as bait. By this approach, we identified OFP1 (Ovate Family Protein 1) as one of DLTinteracting proteins from the yeast library prepared using mixed rice tissues. Full length OFP1 prey vector (OFP1-AD) was further constructed and the interaction was confirmed in yeast (Figure 1A). In addition, we found that when OFP1 was used as both bait and prey, the interaction also occurred (Figure 1A), suggesting that OFP1 may form homo-dimer or oligomer to function.

\section{Subcellular Localization of OFP1 and Verification of the Interaction in Plant Cells}

OFP1 contains both the putative NLS (nuclear localization signal) and NES (nuclear export signal) (Figure 1B). To analyze the protein subcellular localization, we fused the protein with GFP and introduced the corresponding vector into rice protoplasts. Consistently, microscopic observation revealed that OFP1-GFP fusion proteins were localized in both cytoplasm and nucleus, and can be co-localized with DLT-RFP distributed in nucleus (Figure 1C), supporting the possibility that they may interact in plant cells.

We then further verified their interactions by BiFC analysis in rice protoplasts. Different combinations of the proteins fused with either C-terminal or N-terminal of the split Venus YFP (VC or VN) were introduced into the rice protoplast cells, and the reconstituted fluorescence signals were detected using a laser confocal microscope. While as negative controls no obvious signal was detected between any of the fusion proteins and the corresponding empty split YFP partial protein, we observed the strong interaction between VC-OFP1 and VN-DLT in nucleus (Figure 1D). Interestingly, the interacting signals of both protein pairs were mostly spotted in punctate-structures, reminiscent of nuclear bodies which could be formed due to the assembling and spatial-specific distribution of protein complex (Figure 1D). This distinct pattern also indicated that the fluorescence was not false-positive signal and the interaction was indeed occurred. In addition, we also detected the clear interaction between OFP1 itself (Figure 1E). Notably, in most observed cells, the interacting signals were diffusely distributed in part of cells, which appeared to be mainly in cytoplasm, with unclear reason (Figure 1E).

\section{Molecular Characterization of OFP1}

According to rice genome annotation project $^{2}$, OFP1 (LOC_Os01g12690) is intronless and contains 1185-bp coding

\footnotetext{
${ }^{2}$ http://rice.plantbiology.msu.edu
} 
A

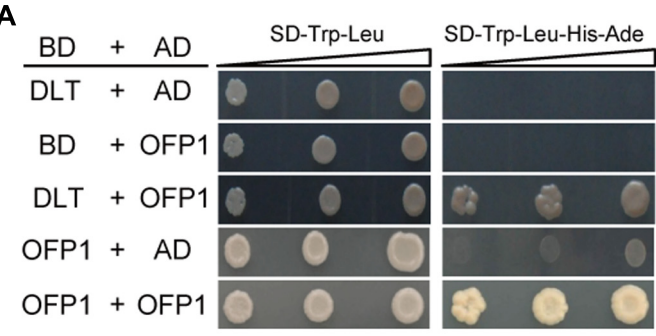

B
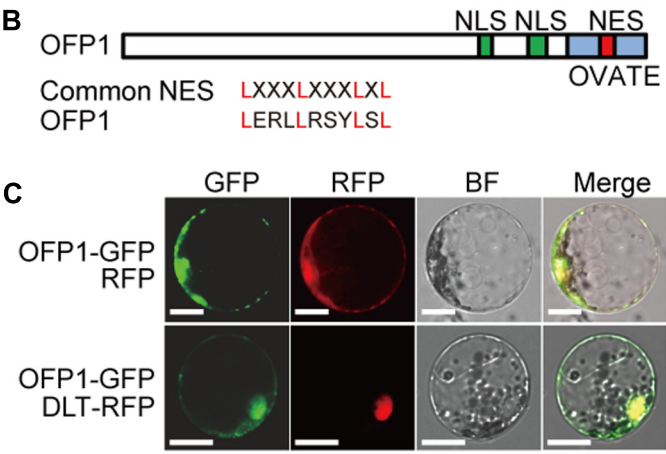

D
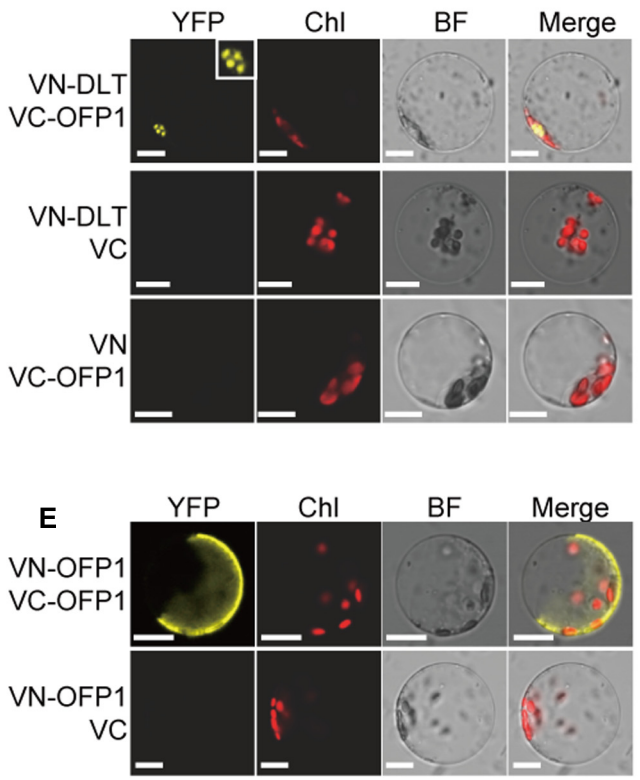

FIGURE 1 | OFP1 interacts with DLT and itself in yeast and plant cells. (A) Yeast two-hybrid analyses of the indicated proteins on minimal synthetic defined media containing -Leu/-Trp or -Leu/-Trp/-His/-Ade dropout supplements. (B) Schematic diagram of OFP1 protein with NES, NLS, and OVATE domain indicated. Alignment of the putative NES with the common NES sequence was shown. (C) Subcellular co-localization of OFP1-GFP with RFP or DLT-RFP. BF, bright field. Scale bars $=10 \mu \mathrm{m}$. (D,E) BiFC analysis of the interactions between the indicated proteins. Nucleus was magnified to show the punctate structure. Chl, chloroplast fluorescence; BF, bright field. Scale bars $=10 \mu \mathrm{m}$.

sequence, 44-bp 5'-UTR and 244-bp 3'-UTR (Figure 2A). We analyzed the gene expression pattern by qRT-PCR, and found $O F P 1$ was ubiquitously expressed in various rice tissues, preferentially higher in young panicles (Figure 2B). We also evaluated the gene promoter activity using GUS reporter and successfully detected the signals in various tissues of OFP1p:GUS transgenic plants, but mainly at young stages of the tissues (Figure 2C).

\section{Knock-out ofp1 Mutants and ofp1 ofp2 Double Mutants Showed No Obvious Morphological Phenotype}

We produced the loss-of-function mutants of OFP1 by CRISPR/Cas9 gene-editing technology. A segment located at the $5^{\prime}$-end of the gene coding sequence was targeted for the editing, and a number of independent homozygous mutant lines were obtained. Two distinct alleles, ofp 1-1 and ofp 1-2, were selected for the further analysis. These alleles contained 35- and 65-bp deletions around the initial codon respectively, leading to the frameshift afterward of the sequence, suggesting that both are knockout mutants (Figure 2A). However, we failed to observe the morphological difference between ofp 1 mutants and WT at both seedling stage and mature stage (Figure 2D). Both the mutants also exhibited indistinguishable BR sensitivity compared with the WT in lamina bending assay (Figure 2E). We further generated ofp 1 ofp 2 double mutant by similar strategy, yet the homozygous mutant plants still showed no obviously phenotypes under normal growth conditions as well as unaltered BR sensitivity in lamina bending assay (Figures 2F,G).

\section{OFP1 Transgenic Plants Exhibit Significantly Altered Plant Architecture and Grain Morphology}

There are 33 OFPs in rice, and different OFP members could function with high redundancy, as strongly suggested in Arabidopsis (Wang et al., 2011; Liu et al., 2014). In this case, transgenic analysis was generally used to analyze the protein function. To this end, the whole OFP1 gene containing 2-kb upstream promoter and coding region was introduced into either Dongjing (DJ) or Zhonghua 11 (ZH11), two different Japonica WTs. Strikingly, although with different extents due to the various gene expression levels, most of the transgenic plants (OFP1-OE, designated as 10 for short in Figures) showed obvious phenotypes in both WT backgrounds (Figure 3). Unless specified, we majorly used transgenic lines in DJ background for our analysis, since we obtained the stable homozygous lines at the first place.

OFP1-OE had narrow leaves, enlarged leaf angles, decreased plant height, reduced tiller number, and long and narrow seeds (Figures 3A-F,H). The grain morphology was remarkably changed due to the greatly increase of the length/width ratio (Figures 3C,E,F). The severe lines are sterile, and have roll leaves and significantly decreased tiller numbers (Figure $\mathbf{3 H}$ ). Most of these phenotypes, including the typical BR-enhanced phenotypes, largely resembled $D L T$-overexpression plants (Do), featured with the significantly narrowed leaves and seeds which 

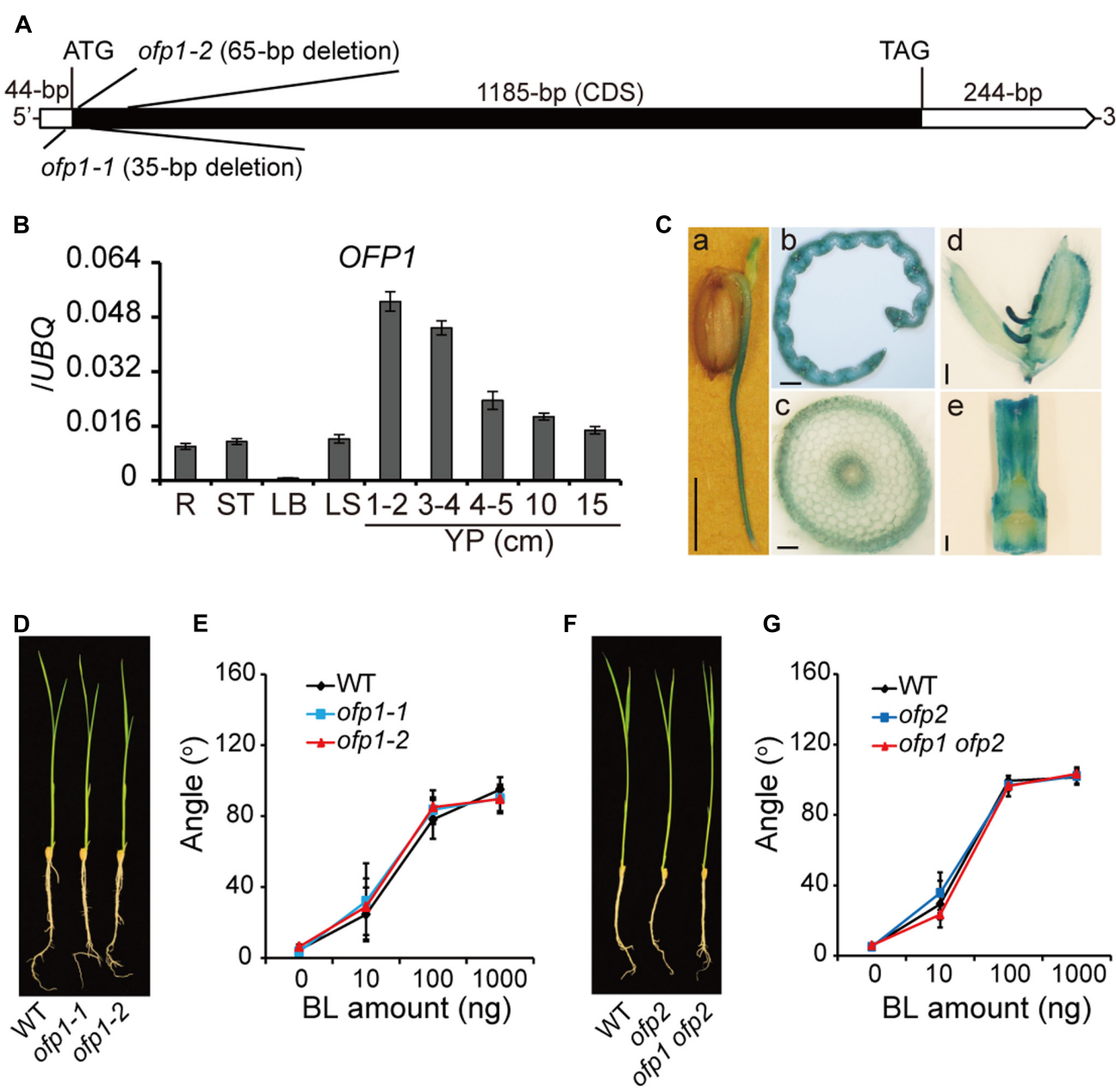

FIGURE 2 | Characterization of OFP1 expression and the ofp1 knockout mutants. (A) Schematic diagram of OFP1 gene structure. UTR and coding sequence were indicated. Mutation sites of ofp1 alleles produced by CRISPR/CAS9 technology were shown. (B) Relative expression levels of OFP1 in various rice tissues tested by qRT-PCR. R, root; ST, stem; LB, leaf blade; LS, leaf sheath; YP, young panicle. Numbers indicate the panicle length (cm). UBQ gene was used as internal reference. $n=3$, bar = SD. (C) OFP1 promoter activity analysis in various tissues evaluated by GUS reporter. (a) coleoptile and root on germinated seed; (b) transverse section of young leaf blade; (c) transverse section of root; (d) spikelet; (e) internode. Scale bars = $5 \mathrm{~mm}$ in (a), $100 \mu \mathrm{m}$ in (b, c), $1 \mathrm{~mm}$ in (d, e). (D) Seedling morphology of ofp1 knockout mutants. (E) Lamina bending analysis of the plants in (D) in response to various amount of BL. $n=10$, bar = SD. (F) Seedling morphology of ofp2 and ofp1 ofp2 knockout mutants. (G) Lamina bending analysis of the plants in (F) in response to various amount of BL. $n=10$, bar $=S D$.

could be, at least partially, BR-independent (Tong et al., 2012). Severities of the phenotypes were obviously consistent with the gene expression levels in the transgenic plants (Figures $3 \mathrm{~A}-\mathbf{I}$ ), suggesting the involvement of OFP1 in controlling these phenotypes. However, we surprisingly found that the gene was remarkably over-expressed in the positive transgenic plants (Figures 3G,I). While the reason is unclear, one possibility is that there exists additional suppressor elements outside the selected promoter region.

\section{OFP1 Enhanced BR Responses and Required DLT to Regulate Plant Architecture}

We further tested the BR sensitivity of the plants by lamina inclination assay. OFP1-OE showed increased leaf angles than
WT either with or without BR (Figures 4 A,B). To exclude the possibility that the enhanced leaf angles were caused by increased BR levels, we analyzed the expression of BR synthetic genes including $D 2, D 11$, and DWARF and found that all of them have markedly decreased expression in OFP1-OE (Figure 4C), similar to that in Do plants (Tong et al., 2012). This result also implied that the BR synthesis was inhibited by enhanced BR responses in a feedback manner in OFP1-OE. Combined with the phenotypes, these results demonstrated that OFP1-OE plants have activated $\mathrm{BR}$ responses.

To further analyze the OFP1 functions, we introduced OFP1 into $d 2$, a BR synthesis mutant (Hong et al., 2003), by transgenic method, and found that 22 OFP1-OE showed obviously enlarged leaf angles and decreased grain width compared with $d 2$ (Figures 4D-F). We also introduced OFP1 into dlt mutant, and found dlt OFP1-OE showed similar morphology as $d l t$, 
A

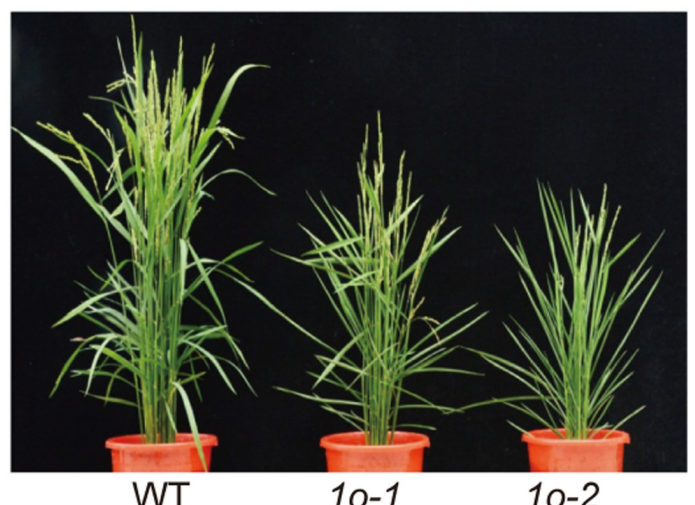

D

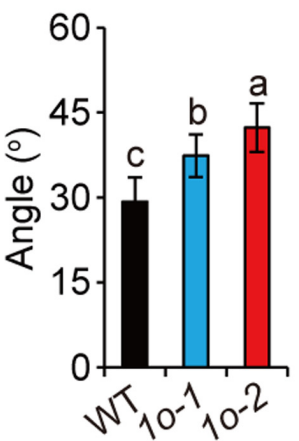

B

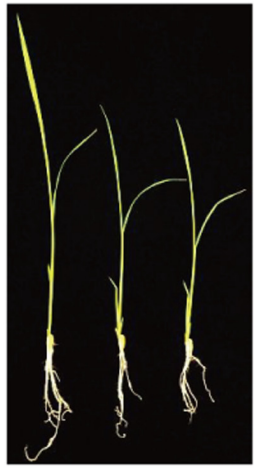

WT 10-1 10-2

E

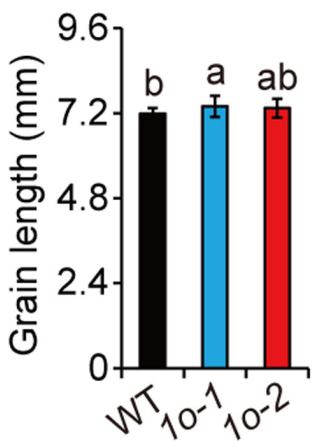

F

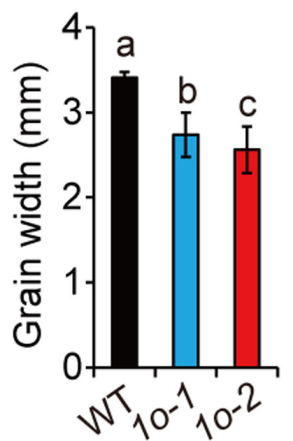

C
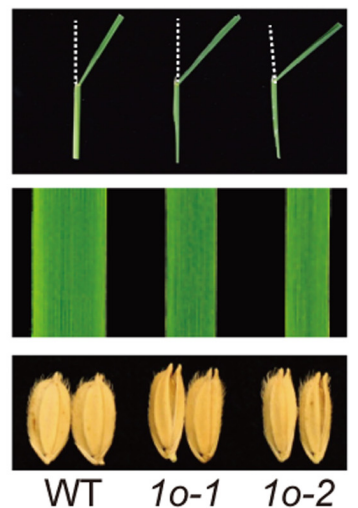

G



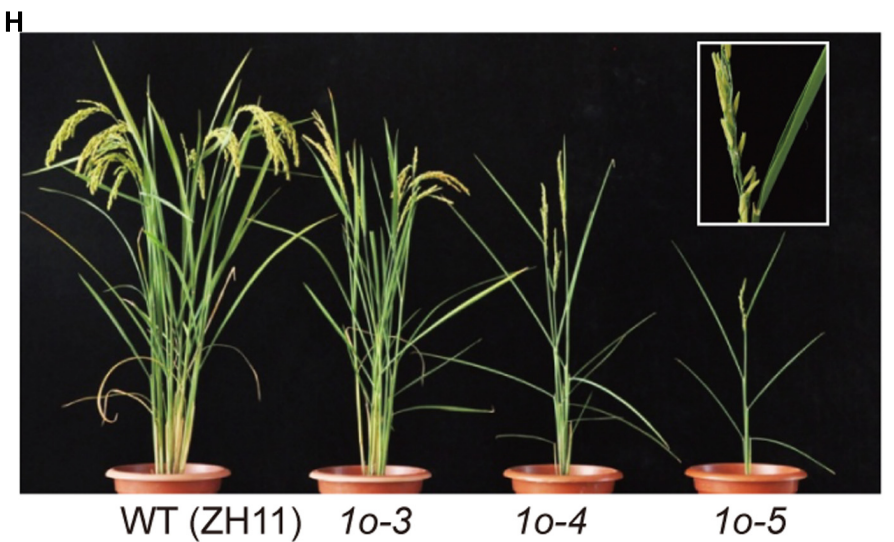

I

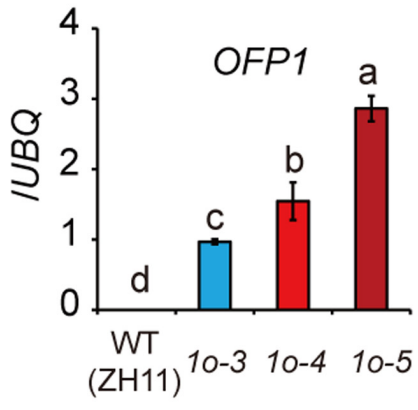

FIGURE 3 | Phenotype analysis of OFP1 overexpression plants. (A) Gross morphology of two representing OFP1 overexpression plants in DJ background, designated as 10-1 and 10-2 for short, at the reproductive stage. (B) Seedling morphology of 10-1 and 10-2 compared with WT. (C) Detailed comparison of leaf angle, leaf width, and grain morphology. Statistical data of the leaf angle (D), grain length (E), and grain width (F) of the plants. $n=12$ in (D), $n=20$ in (E,F), bar $=$ SD. Different letters above the columns indicate statistically significant differences between groups (Fisher's LSD, $p<0.05$ ). (G) Relative OFP1 expression in the plants tested by qRT-PCR. UBQ gene was used as internal reference. $n=3$, bar $=$ SD. Different letters above the columns indicate statistically significant differences between groups (Fisher's LSD, $p<0.05$ ). (H) Phenotypes of three representing OFP1 overexpression plants in ZH11 background, designated as 10-3, 10-4, and 10-5 for short. Enlarged picture showed the development of sterile seeds and roll leaves in the severe line. (I) Relative OFP1 expression in the plants tested by qRT-PCR. UBQ gene was used as internal reference. $n=3$, bar $=$ SD. Different letters above the columns indicate statistically significant differences between groups (Fisher's LSD, $p<0.05$ ).

suggesting that OFP1 required DLT to regulate plant architecture (Figure 4G). It should be noted that OFP1-OE can obviously suppress the grain width of $d l$ mutant (Figures $4 \mathbf{G}, \mathbf{H}$ ), demonstrating that the protein is functional in the transgenic plant. Considering BR has relatively minor roles in regulating grain width (Tanabe et al., 2005), this result indicated that OFP1 and DLT have differential functional relationship in different tissues or biological processes. Taken together, these results strongly suggested that OFP1 positively regulates BR responses. 


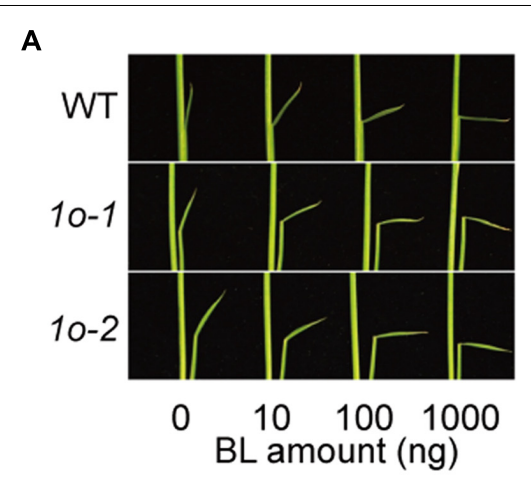

D

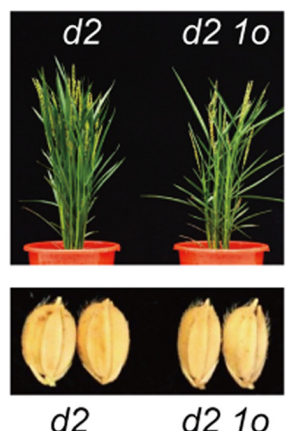

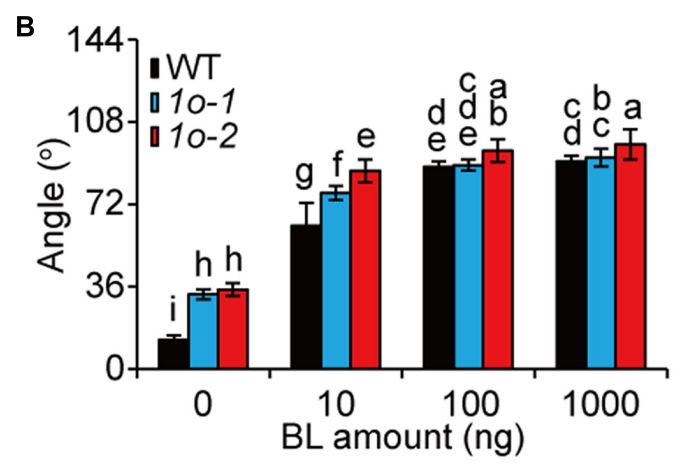

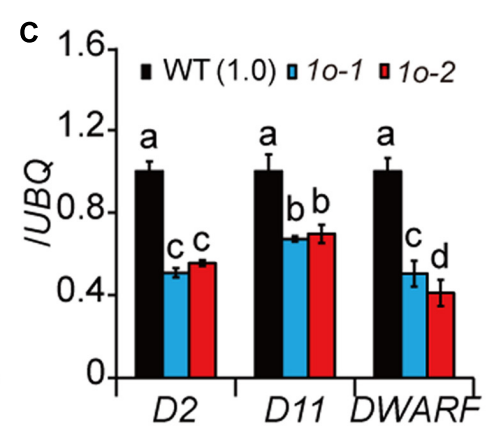

FIGURE 4 | OFP1 overexpression plants have enhanced BR responses. (A,B) Lamina bending analysis of the BR sensitivity of 10-1 and 10-2. Statistical data were shown in (B). $n=10$, bar = SD. Different letters above the columns indicate statistically significant differences between groups (Fisher's LSD, $p<0.05)$. (C) Relative expression of the BR synthetic genes in 10-1 and 10-2. Expression in WT was set as 1.0. UBQ gene was used as internal reference. $n=3$, bar $=$ SD. Different letters above the columns indicate statistically significant differences between groups (Fisher's LSD, $p<0.05$ ). OFP1 overexpression in $d 2$ background (D) and statistical data of the leaf angle (E) and grain width of the plants (F). $n=5$ in (E), $n=20$ in (F), bar $=\mathrm{SD},{ }^{* *} p<0.01$ in Student's $t$-test. OFP1 overexpression in dlt background (G) and statistical data of the grain width of the plants $\mathbf{( H )} . n=20$, bar $=\mathrm{SD}$. Different letters above the columns indicate statistically significant differences between groups (Fisher's LSD, $p<0.05$ ).

\section{BR Promotes OFP1 Expression and OsBZR1 Binds to OFP1 Promoter}

We have performed RNA-seq analysis to compare the differentially expressed genes between high $\mathrm{BL}$ and low $\mathrm{BL}$ treated rice plants. In our available data, we found OFP1 was differentially over-expressed in $10^{-6} \mathrm{M} \mathrm{BL}$ treated rice seedlings, but not in $10^{-9} \mathrm{M}$ BL treated materials. To confirm this result, we treated WT plants using various concentrations of $\mathrm{BL}$, and then analyzed the expression of OFP1 by qRT-PCR. Consistently, the results showed that OFP1 was markedly induced by application of BR above $10^{-7} \mathrm{M}$, but not induced under lower BR concentrations (Figure 5A). In addition, our available ChIP-seq data using OsBZR1 antibody enriched OsBZR1 protein on the promoter region of OFP1 with 3.28fold increase in WT rice seedlings. Accordingly, we found OFP1 promoter contains multiple cis-elements that have been suggested to be bound by BZR1 (Figure 5B) (Sun et al., 2010; Yu et al., 2011). The association between OsBZR1 and the OFP1 promoter was further confirmed by ChIP-qPCR (Figure 5C). Compared with the samples with no antibody supplemented, OsBZR1 antibody can significantly pull down the
DNA segments containing the putative BZR1 binding elements (Figure 5C).

\section{OFP1 Is Involved in BR Inhibition of GA Synthesis and Plant Growth}

In Arabidopsis, it was shown that AtOFP1 directly suppresses GA synthetic gene to repress plant growth (Wang et al., 2007). Our previous study also suggested that high BR levels or enhanced BR signaling will repress GA level to inhibit plant growth in rice (Tong et al., 2014). The specific induction of OFP1 by high BR (Figure 5A) and the strongly repressed growth of OFP1-OE plants (Figures 3A,B) prompted us to test whether OFP1 was involved in this process. Indeed, we found GA3ox-2 and GA20ox-2, two GA synthetic genes, had decreased expression, whereas GA2ox-3, the GA inactivation gene, had increased expression in OFP1-OE (Figure 6A). Accordingly, $\mathrm{GA}_{1}$, the major bioactive GA form in young seedlings, was significantly decreased in the plants (Figure 6B), consistent with their decreased seedling height (Figure 3B). Actually, all the GA forms quantified were decreased in OFP1-OE plants (Figure 6B), reminiscent of the hormonal profiles obtained 


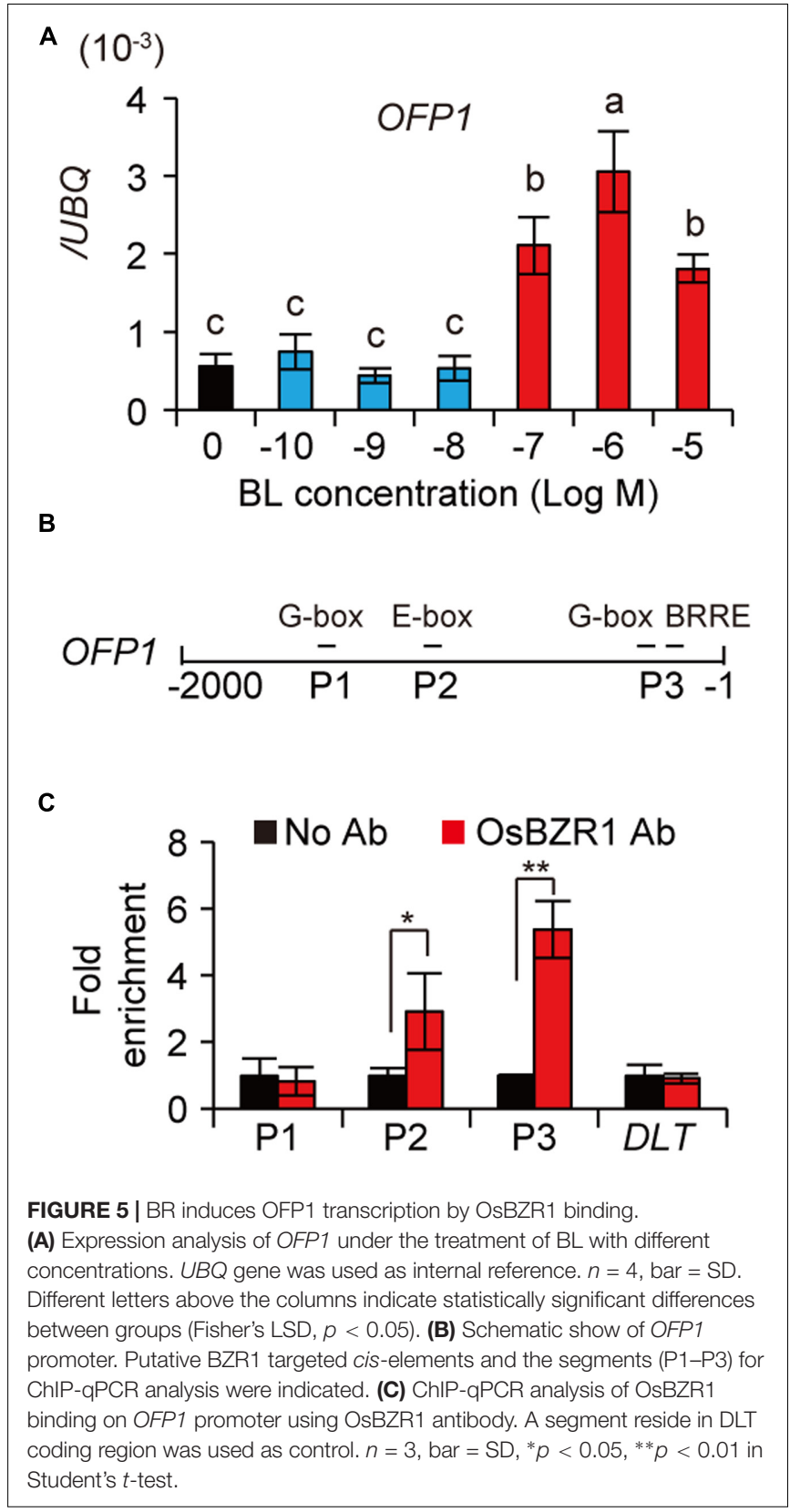

from high-BL-treated plants as shown previously (Tong et al., 2014). These results indicated that OFP1 is involved in the BLinduced GA repression and growth inhibition, depending on BR concentrations or plant tissues.

\section{OFP1 Protein Stability Was Regulated by BR and Development Stages}

To test whether OFP1 was regulated at protein level, we fused the coding sequence with Flag tag and introduced into the WT under 35S promoter (OFP1-Flag-OE, designated as 1 Fo for short in Figures). We obtained a collection of the overexpression plants which showed similar phenotypes as above described OFP1-OE plants, including enlarged leaf angle, reduced plant height, and altered grain shape (Figure 7A). Immunoblotting analysis using Flag antibody can detect the protein expression in the corresponding plants, demonstrating the fusion proteins are functional (Figure 7B). We treated the plants with different concentrations of $\mathrm{BL}$, and found the protein was gradually accumulated (Figure $7 \mathrm{C}$ ). We also tested the OFP1 protein in different leaves representing the different developmental stages, and revealed that the protein was highly accumulated in young leaves, but gradually decreased in old leaves (Figure 7D). These results suggested that OFP1 was dynamically regulated by hormone levels and development stages.

\section{GSK2 Interacts with OFP1 and Regulates OFP1 Stability}

GSK3-like kinases, as the central negative regulators of BR signaling, can interact with many transcriptional factors to modulate their stability and activity (Youn and Kim, 2015). The promotion of OFP1 stability by BR prompted us to test whether GSK2 can directly interact with OFP1 to regulate this process. Indeed, yeast two-hybrid analysis revealed the obviously interaction between GSK2 bait and OFP1 prey (Figure 8A). Surprisingly, we can rarely or only weakly observe the interaction between GSK2 and OFP1 by BiFC analysis in rice protoplasts. One possibility is that the interaction is unstable in rice, considering GSK2 might promote OFP1 degradation. Alternatively, we adopted split-luciferase complementation assay to analyze their interaction in tobacco leaves (Figure $\mathbf{8 B}$ ). When the GSK2 fused with N-terminal of luciferase protein (GSK2NLuc) was co-expressed with the OFP1 fused with C-terminal of luciferase protein (CLuc-OFP1), the interaction signals were evidently detected. No signal was detected when the GSK2-NLuc was co-expressed with the empty CLuc protein (Figure 8B). Thus, GSK2 indeed can interact with OFP1 in plant.

To explore whether GSK2 regulates OFP1 stability, we treated OFP1-Flag-OE plants using bikinin, a specific chemical inhibitor of GSK3-like kinase activity, and analyzed the OFP1 protein level. Compared with the plants without treatment, we found OFP1 was significantly accumulated (Figure $\mathbf{8 C}$ ). Taken together, these results suggested that $\mathrm{BR}$ enhances $\mathrm{OFP} 1$ protein stability by inhibiting GSK2.

\section{DISCUSSION}

Our study identified OFP1 as an additional positive player of BR responses by interacting with GSK2, OsBZR1, and DLT. We proposed that (1) at transcription level, BR induces OFP1 expression through OsBZR1, (2) at protein level, BR enhances OFP1 stability by inactivate GSK2, and (3) OFP1 activity partially relies on the physical interaction with DLT. In addition, our results suggested the novel roles of OsBZR1-induced OFP1 that required for responding to elevated $\mathrm{BR}$.

Functional characterization of OFPs progressed slowly, largely due to the high redundancy among the family members (Wang et al., 2011). To our knowledge, there is no phenotype-evident knockout plants of any OFP members reported in both rice and Arabidopsis. In Arabidopsis, T-DNA insertion ofp mutants 

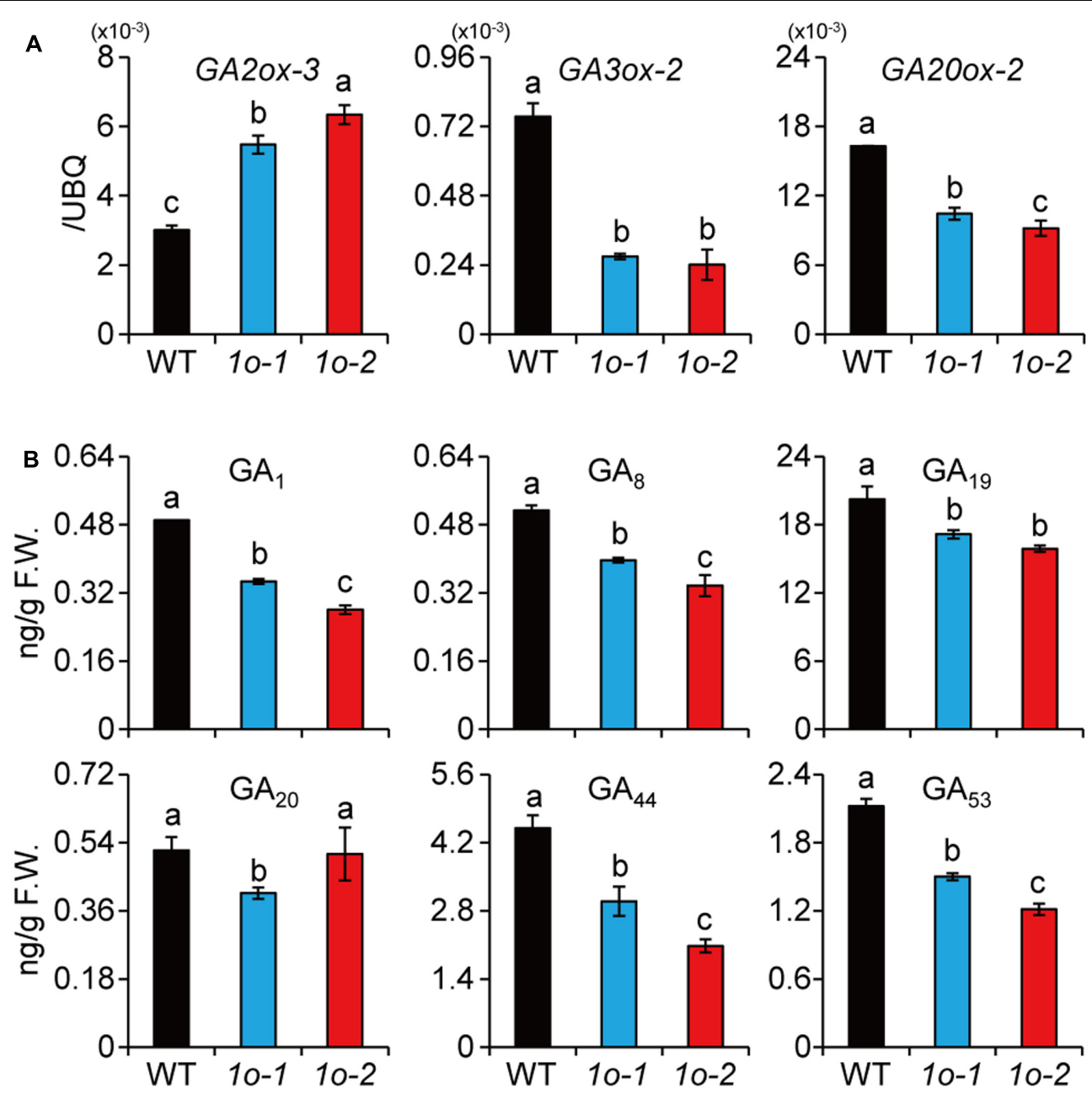

FIGURE 6 | OFP1 overexpression plants have decreased GA synthesis. (A) Relative expression of the GA metabolism genes in 10-1 and 10-2. Expression in WT was set as 1.0. UBQ gene was used as internal reference. $n=3$, bar $=\mathrm{SD}$. Different letters above the columns indicate statistically significant differences between groups (Fisher's LSD, $p<0.05$ ). (B) Quantification of GA levels in 10-1 and 10-2. F.W., fresh weight. $n=3$, bar $=\mathrm{SD}$. Different letters above the columns indicate statistically significant differences between groups (Fisher's LSD, $p<0.05$ ).

including ofp 1, ofp 4, ofp 8, ofp10, ofp 15, and ofp16, all showed no visible phenotype under normal growth condition. In addition, the double mutants including ofp 1 ofp 4 and ofp15 ofp16 also showed no obvious phenotype (Wang et al., 2011). This seemed to be also the case in rice. The ofp 1 knockout mutant, as well as ofp 1 ofp 2 double mutant, didn't produce the expected phenotypes (Figure 2). However, knock-down of OFP8/OFP30 by RNAi strategy indeed led to BR-related phenotypes (Yang et al., 2016). Although it's unclear whether other members were non-specifically targeted, this result at least suggested the native function of OFPs in regulating BR responses. On the other hand, OFP genes tend to be flexibly regulated by various hormones ( $\mathrm{Yu}$ et al., 2015), and overexpression of OFPs usually resulted in obvious morphological changes, suggesting their roles in regulating hormone responses. The extensively interaction between OFPs and KNOX proteins further implied this speculation, considering the crucial roles of KNOXs in regulating hormone balance to maintain meristem activity (Hackbusch et al., 2005; Tsuda et al., 2014). Given the distinct phenotypes of the OFP1 overexpression plants, and the remarkable regulation of OFP1 transcription and protein by BRs, our study should, at least partially, revealed the function of the native protein in plant.

OFP1 function is closely related to DLT, both positively regulate $\mathrm{BR}$ responses. The overexpression plants of the two genes are highly similar. It should be mentioned that although $d l t$ mutant showed decreased tiller number, the severe DLT-overexpression lines could also have decreased tiller number, similar as OFP1-OE. Genetic analysis showed that OFP1 function in regulating plant architecture, but not the grain width, is dependent on DLT (Figure 4). It should be mentioned that the significant regulation of grain width by DLT could be partially independent of BR response, since the typical BR mutants have little alteration of grain width (Tanabe et al., 2005). Accordingly, OFP1 also has marked effect on grain width, further implying the functional relevance between OFP1 and DLT. Notably, the 
A

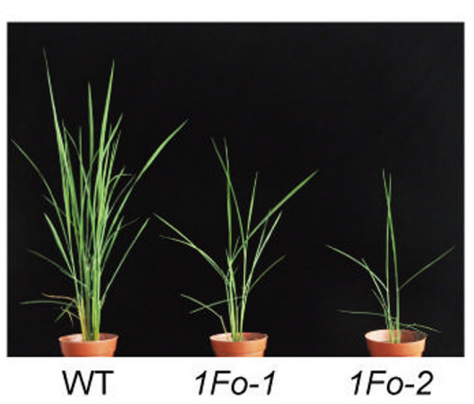

c
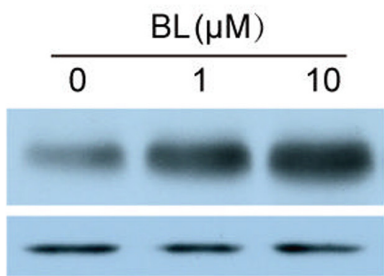

B

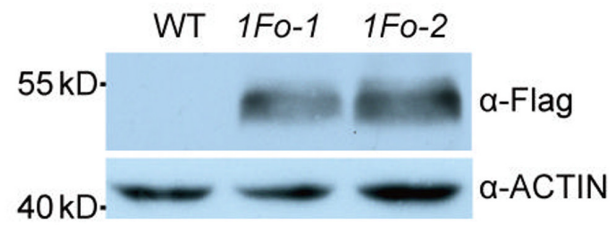

D

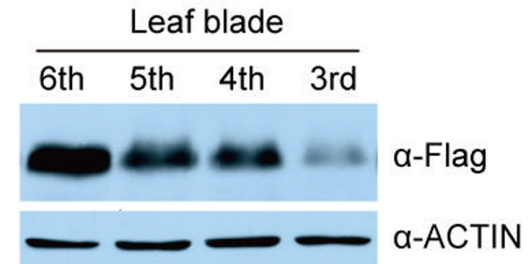

FIGURE 7 | OFP1 protein was regulated by BR and developmental stage. (A) Gross morphology of two representative OFP1-Flag overexpression plants, designated as 1 FO-1 and 1FO-2 for short. (B) Immunoblotting analysis of OFP1-Flag fusion proteins in the indicated rice plants. Rice ACTIN protein was blotted as loading reference. (C) Effects of $\mathrm{BL}$ on the protein stability of OFP1-Flag in 1FO-2. Rice ACTIN protein was blotted as loading reference. (D) Immunoblotting analysis of OFP1-Flag fusion proteins in different leaf blades in 1FO-2. Rice ACTIN protein was blotted as loading reference.

A

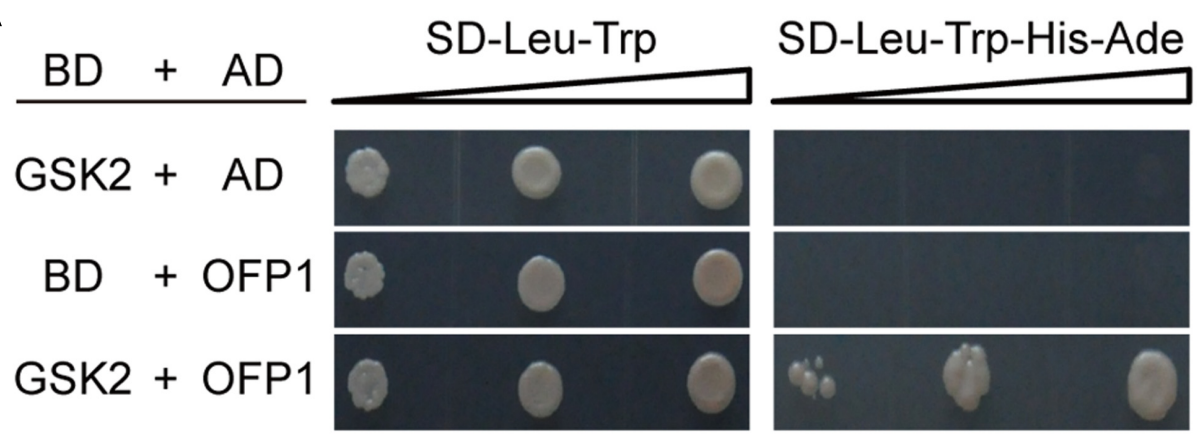

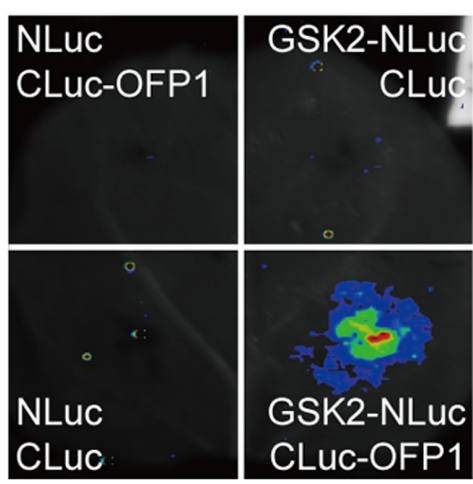

C

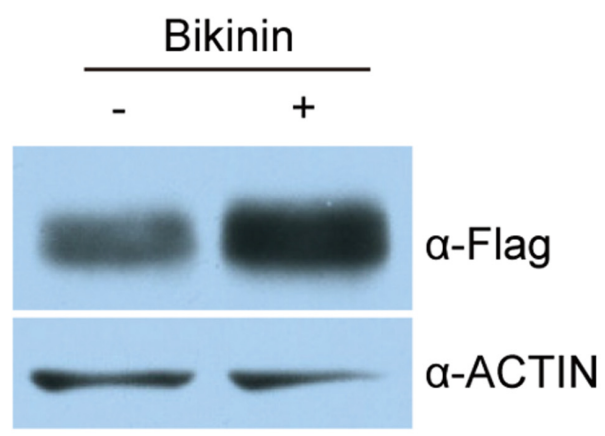

FIGURE 8 | GSK2 interacts with OFP1 and regulates OFP1 stability. (A) Yeast two-hybrid analysis of the interaction between GSK2 and OFP1. (B) Split luciferase complementation analysis of the interaction between GSK2 and OFP1. (C) Effects of bikinin, the GSK3-like kinase inhibitor, on the protein stability of OFP1-Flag in 1Fo-2. Rice ACTIN protein was blotted as loading reference. 
regulation of grain shape (length/width ratio) by OFP1 and DLT in rice was somewhat reminiscent of OVATE or OVATE-like gene in regulating the fruit shape in both tomato and pepper (Liu et al., 2002; Tsaballa et al., 2011), potentially suggesting a general role of OFPs in regulating seed development. A bold speculation is that OFPs could have indispensable regulatory roles in plant growth and development, thus plant has developed highly redundant system to maintain the normal function of the family.

Although BRs were known to be a class of growth-promoting hormones, it was found that most BR-enhanced plants didn't have increased plant height, and many of them actually exhibited decreased plant height in rice (Wang et al., 2008; Li et al., 2009; Tanaka et al., 2009; Tong et al., 2012). Consistently, exogenous high BR treatment also inhibits plant seedling growth. We previously discovered that this is because high BR or increased BR signaling turned to repress GA synthesis (Tong et al., 2014; Che et al., 2015). These observations suggested that BR balance is very important for plant growth. In this study, we revealed that OFP1 should play a role in this process. Overexpression of AtOFP1 in Arabidopsis, or OsOFP1 or OsOFP2 or OsOFP8 in rice, all leads to greatly reduced plant height (Wang et al., 2007; Schmitz et al., 2015; Yang et al., 2016). AtOFP1 directly targets GA20ox-1 to suppress GA synthesis, and OsOFP2 was also suggested to regulate GA20ox-7 to suppress GA synthesis. Measurement of GA contents in rice $O F P 1$ overexpression plants demonstrated the decreased GA levels of basically all GA forms (Figure 6B). Notably, this profile is very similar to that in the high BR treated plants, which also contained decreased levels of basically all quantified GA forms (Tong et al., 2014). Actually, the OFP1 overexpression plants largely mimicked the high BR treated WT plants, including enlarged leaf angles and decreased plant height. Altogether, OFP1 should be involved in inhibition of GA levels and plant growth elicited by either high BR levels or elevated BR signaling.

\section{REFERENCES}

Bart, R., Chern, M., Park, C. J., Bartley, L., and Ronald, P. C. (2006). A novel system for gene silencing using siRNAs in rice leaf and stem-derived protoplasts. Plant Methods 2:13. doi: 10.1186/1746-4811-2-13

Che, R., Tong, H., Shi, B., Liu, Y., Fang, S., Liu, D., et al. (2015). Control of grain size and rice yield by GL2-mediated brassinosteroid responses. Nat. Plants 2:15195. doi: 10.1038/nplants.2015.195

Chen, H., Zou, Y., Shang, Y., Lin, H., Wang, Y., Cai, R., et al. (2008). Firefly luciferase complementation imaging assay for protein-protein interactions in plants. Plant Physiol. 146, 368-376. doi: 10.1104/pp.107.111740

Chen, M. L., Fu, X. M., Liu, J. Q., Ye, T. T., Hou, S. Y., Huang, Y. Q., et al. (2012). Highly sensitive and quantitative profiling of acidic phytohormones using derivatization approach coupled with nano-LC-ESI-Q-TOF-MS analysis. J. Chromatogr. B Analyt. Technol. Biomed. Life Sci. 905, 67-74. doi: 10.1016/j. jchromb.2012.08.005

Divi, U. K., and Krishna, P. (2009). Brassinosteroid: a biotechnological target for enhancing crop yield and stress tolerance. New Biotechnol. 26, 131-136. doi: $10.1016 /$ j.nbt.2009.07.006

Gruszka, D. (2013). The brassinosteroid signaling pathway-new key players and interconnections with other signaling networks crucial for plant development and stress tolerance. Int. J. Mol. Sci. 14, 8740-8774. doi: 10.3390/ijms14058740

Gudesblat, G. E., Schneider-Pizon, J., Betti, C., Mayerhofer, J., Vanhoutte, I., van Dongen, W., et al. (2012). SPEECHLESS integrates brassinosteroid and stomata signalling pathways. Nat. Cell Biol. 14, 548-554. doi: 10.1038/ncb2471

\section{CONCLUSION}

Elevated BR signaling will induce OFP1 expression by OsBZR1, and also promote protein stability by inhibiting GSK2, leading to activation of OFP1, which interacts with DLT factors and targets downstream genes, including GA metabolism genes, to regulate plant architecture and grain morphology in rice.

\section{AUTHOR CONTRIBUTIONS}

YX and DL performed the study with the assistance of GZ and HT. HT, YX, and CC analyzed the data and wrote the manuscript. HT and CC conceived and supervised the study.

\section{ACKNOWLEDGMENTS}

We thank Prof. Jiayang Li and Prof. Jianmin Zhou (Institute of Genetics and Developmental Biology) for providing BiFC and split-LUC vectors, respectively, and Prof. Yaoguang Liu (South China Agricultural University) for providing CRISPR/Cas9 tools. This work was supported by National Natural Science Foundation (Nos. 91435106 and 91335203) and CAAS Elite Youth Program start-up funding (to HT).

\section{SUPPLEMENTARY MATERIAL}

The Supplementary Material for this article can be found online at: http://journal.frontiersin.org/article/10.3389/fpls.2017.01698/ full\#supplementary-material

Hackbusch, J., Richter, K., Muller, J., Salamini, F., and Uhrig, J. F. (2005). A central role of Arabidopsis thaliana ovate family proteins in networking and subcellular localization of 3-aa loop extension homeodomain proteins. Proc. Natl. Acad. Sci. U.S.A. 102, 4908-4912. doi: 10.1073/pnas.050118 1102

Hao, J. J., Yin, Y. H., and Fei, S. Z. (2013). Brassinosteroid signaling network: implications on yield and stress tolerance. Plant Cell Rep. 32, 1017-1030. doi: 10.1007/s00299-013-1438-x

He, J. X., Gendron, J. M., Yang, Y., Li, J., and Wang, Z. Y. (2002). The GSK3-like kinase BIN2 phosphorylates and destabilizes BZR1, a positive regulator of the brassinosteroid signaling pathway in Arabidopsis. Proc. Natl. Acad. Sci. U.S.A. 99, 10185-10190. doi: 10.1073/pnas. 152342599

Hirano, K., Yoshida, H., Aya, K., Kawamura, M., Hayashi, M., Hobo, T., et al. (2017). Small organ size 1 and small organ size 2/dwarf and low tillering form a complex to integrate auxin and brassinosteroid signaling in rice. Mol. Plant 10, 590-604. doi: 10.1016/j.molp.2016.12.013

Hong, Z., Ueguchi-Tanaka, M., Umemura, K., Uozu, S., Fujioka, S., Takatsuto, S., et al. (2003). A rice brassinosteroid-deficient mutant, ebisu dwarf (d2), is caused by a loss of function of a new member of cytochrome P450. Plant Cell 15, 2900-2910. doi: 10.1105/tpc.014712

Kim, T. W., Michniewicz, M., Bergmann, D. C., and Wang, Z. Y. (2012). Brassinosteroid regulates stomatal development by GSK3-mediated inhibition of a MAPK pathway. Nature 482, 419-422. doi: 10.1038/nature 10794 
Li, D., Wang, L., Wang, M., Xu, Y. Y., Luo, W., Liu, Y. J., et al. (2009). Engineering OsBAK1 gene as a molecular tool to improve rice architecture for high yield. Plant Biotechnol. J. 7, 791-806. doi: 10.1111/j.1467-7652.2009. 00444.x

Liu, D., Sun, W., Yuan, Y. W., Zhang, N., Hayward, A., Liu, Y. L., et al. (2014). Phylogenetic analyses provide the first insights into the evolution of OVATE family proteins in land plants. Ann. Bot. 113, 1219-1233. doi: 10.1093/aob/ mcu061

Liu, J., Van Eck, J., Cong, B., and Tanksley, S. D. (2002). A new class of regulatory genes underlying the cause of pear-shaped tomato fruit. Proc. Natl. Acad. Sci. U.S.A. 99, 13302-13306. doi: 10.1073/pnas.162485999

Ma, X., and Liu, Y. G. (2016). CRISPR/Cas9-based multiplex genome editing in monocot and dicot plants. Curr. Protoc. Mol. Biol. 115, 31.6.1-31.6.21. doi: $10.1002 / \mathrm{cpmb} .10$

Morinaka, Y., Sakamoto, T., Inukai, Y., Agetsuma, M., Kitano, H., Ashikari, M., et al. (2006). Morphological alteration caused by brassinosteroid insensitivity increases the biomass and grain production of rice. Plant Physiol. 141, 924-931. doi: 10.1104/pp.106.077081

Nolan, T. M., Brennan, B., Yang, M. R., Chen, J. N., Zhang, M. C., Li, Z. H., et al. (2017). Selective autophagy of BES1 mediated by DSK2 balances plant growth and survival. Dev. Cell 41, 33-46. doi: 10.1016/j.devcel.2017. 03.013

Qiao, S., Sun, S., Wang, L., Wu, Z., Li, C., Li, X., et al. (2017). The RLA1/SMOS1 transcription factor functions with OsBZR1 to regulate brassinosteroid signaling and rice architecture. Plant Cell 29, 292-309. doi: 10.1105/tpc.16. 00611

Sakamoto, T., Morinaka, Y., Ohnishi, T., Sunohara, H., Fujioka, S., UeguchiTanaka, M., et al. (2006). Erect leaves caused by brassinosteroid deficiency increase biomass production and grain yield in rice. Nat. Biotechnol. 24, 105-109. doi: 10.1038/nbt1173

Schmitz, A. J., Begcy, K., Sarath, G., and Walia, H. (2015). Rice Ovate Family Protein 2 (OFP2) alters hormonal homeostasis and vasculature development. Plant Sci. 241, 177-188. doi: 10.1016/j.plantsci.2015.10.011

Singh, A. P., and Savaldi-Goldstein, S. (2015). Growth control: brassinosteroid activity gets context. J. Exp. Bot. 66, 1123-1132. doi: 10.1093/jxb/ erv026

Sparkes, I. A., Runions, J., Kearns, A., and Hawes, C. (2006). Rapid, transient expression of fluorescent fusion proteins in tobacco plants and generation of stably transformed plants. Nat. Protoc. 1, 2019-2025. doi: 10.1038/nprot. 2006.286

Sun, Y., Fan, X. Y., Cao, D. M., Tang, W. Q., He, K., Zhu, J. Y., et al. (2010). Integration of brassinosteroid signal transduction with the transcription network for plant growth regulation in Arabidopsis. Dev. Cell 19, 765-777. doi: 10.1016/j.devcel.2010.10.010

Tanabe, S., Ashikari, M., Fujioka, S., Takatsuto, S., Yoshida, S., Yano, M., et al. (2005). A novel cytochrome P450 is implicated in brassinosteroid biosynthesis via the characterization of a rice dwarf mutant, dwarf11, with reduced seed length. Plant Cell 17, 776-790. doi: 10.1105/tpc.104. 024950

Tanaka, A., Nakagawa, H., Tomita, C., Shimatani, Z., Ohtake, M., Nomura, T., et al. (2009). BRASSINOSTEROID UPREGULATED1, encoding a Helix-LoopHelix protein, is a novel gene involved in brassinosteroid signaling and controls bending of the lamina joint in rice. Plant Physiol. 151, 669-680. doi: 10.1104/ pp.109.140806

Tong, H., and Chu, C. (2017). Physiological analysis of brassinosteroid responses and sensitivity in rice. Methods Mol. Biol. 1564, 23-29. doi: 10.1007/978-14939-6813-8_3

Tong, H., Xiao, Y., Liu, D., Gao, S., Liu, L., Yin, Y., et al. (2014). Brassinosteroid regulates cell elongation by modulating gibberellin metabolism in rice. Plant Cell 26, 4376-4393. doi: 10.1105/tpc.114.132092

Tong, H. N., and Chu, C. C. (2012). Brassinosteroid signaling and application in rice. J. Genet. Genomics 39, 3-9. doi: 10.1016/j.jgg.2011.12.001

Tong, H. N., and Chu, C. C. (2016). Reply: brassinosteroid regulates gibberellin synthesis to promote cell elongation in rice: critical comments on Ross and Quittenden's Letter. Plant Cell 28, 833-835. doi: 10.1105/tpc.16. 00123

Tong, H. N., Jin, Y., Liu, W. B., Li, F., Fang, J., Yin, Y. H., et al. (2009). DWARF AND LOW-TILLERING, a new member of the GRAS family, plays positive roles in brassinosteroid signaling in rice. Plant J. 58, 803-816. doi: 10.1111/j. 1365-313X.2009.03825.x

Tong, H. N., Liu, L. C., Jin, Y., Du, L., Yin, Y. H., Qian, Q., et al. (2012). DWARF AND LOW-TILLERING acts as a direct downstream target of a GSK3/SHAGGY-like kinase to mediate brassinosteroid responses in rice. Plant Cell 24, 2562-2577. doi: 10.1105/tpc.112.097394

Tsaballa, A., Pasentsis, K., Darzentas, N., and Tsaftaris, A. S. (2011). Multiple evidence for the role of an Ovate-like gene in determining fruit shape in pepper. BMC Plant Biol. 11:46. doi: 10.1186/1471-2229-11-46

Tsuda, K., Kurata, N., Ohyanagi, H., and Hake, S. (2014). Genome-wide study of KNOX regulatory network reveals brassinosteroid catabolic genes important for shoot meristem function in rice. Plant Cell 26, 3488-3500. doi: 10.1105/tpc. 114.129122

Unterholzner, S. J., Rozhon, W., Papacek, M., Ciomas, J., Lange, T., Kugler, K. G., et al. (2015). Brassinosteroids are master regulators of gibberellin biosynthesis in Arabidopsis. Plant Cell 27, 2261-2272. doi: 10.1105/tpc.15.00433

Unterholzner, S. J., Rozhon, W., and Poppenberger, B. (2016). Reply: interaction between brassinosteroids and gibberellins: Synthesis or signaling? In Arabidopsis Both! Plant Cell 28, 836-839. doi: 10.1105/tpc.16.00120

Vriet, C., Russinova, E., and Reuzeau, C. (2013). From squalene to brassinolide: the steroid metabolic and signaling pathways across the plant kingdom. Mol. Plant 6, 1738-1757. doi: 10.1093/mp/sst096

Waadt, R., Schmidt, L. K., Lohse, M., Hashimoto, K., Bock, R., and Kudla, J. (2008). Multicolor bimolecular fluorescence complementation reveals simultaneous formation of alternative CBL/CIPK complexes in planta. Plant J. 56, 505-516. doi: 10.1111/j.1365-313X.2008.03612.x

Wang, L., Xu, Y., Zhang, C., Ma, Q., Joo, S. H., Kim, S. K., et al. (2008). OsLIC, a novel CCCH-type zinc finger protein with transcription activation, mediates rice architecture via brassinosteroids signaling. PLOS ONE 3:e3521. doi: 10.1371/journal.pone.0003521

Wang, S., Chang, Y., Guo, J., and Chen, J. G. (2007). Arabidopsis ovate family protein 1 is a transcriptional repressor that suppresses cell elongation. Plant J. 50, 858-872. doi: 10.1111/j.1365-313X.2007.03096.x

Wang, S., Chang, Y., Guo, J., Zeng, Q., Ellis, B. E., and Chen, J. G. (2011). Arabidopsis ovate family proteins, a novel transcriptional repressor family, control multiple aspects of plant growth and development. PLOS ONE 6:e23896. doi: 10.1371/journal.pone.0023896

Yang, C., Shen, W. J., He, Y., Tian, Z. H., and Li, J. X. (2016). OVATE Family Protein 8 positively mediates brassinosteroid signaling through interacting with the GSK3-like kinase in rice. PLOS Genet. 12:e1006118. doi: 10.1371/journal. pgen. 1006118

Yang, M. R., Li, C. X., Cai, Z. Y., Hu, Y. M., Nolan, T., Yu, F. F., et al. (2017). SINAT E3 ligases control the light-mediated stability of the brassinosteroidactivated transcription factor BES1 in Arabidopsis. Dev. Cell 41, 47-58. doi: 10.1016/j.devcel.2017.03.014

Yang, M. R., and Wang, X. L. (2017). Multiple ways of BES1/BZR1 degradation to decode distinct developmental and environmental cues in plants. Mol. Plant 10, 915-917. doi: 10.1016/j.molp.2017.06.005

Yin, Y., Wang, Z. Y., Mora-Garcia, S., Li, J., Yoshida, S., Asami, T., et al. (2002). BES1 accumulates in the nucleus in response to brassinosteroids to regulate gene expression and promote stem elongation. Cell 109, 181-191. doi: 10.1016/ S0092-8674(02)00721-3

Youn, J. H., and Kim, T. W. (2015). Functional insights of plant GSK3-like kinases: multi-taskers in diverse cellular signal transduction pathways. Mol. Plant 8, 552-565. doi: 10.1016/j.molp.2014.12.006

Yu, H., Jiang, W., Liu, Q., Zhang, H., Piao, M., Chen, Z., et al. (2015). Expression pattern and subcellular localization of the ovate protein family in rice. PLOS ONE 10:e0118966. doi: 10.1371/journal.pone.0118966

Yu, X. F., Li, L., Zola, J., Aluru, M., Ye, H. X., Foudree, A., et al. (2011). A brassinosteroid transcriptional network revealed by genome-wide identification of BESI target genes in Arabidopsis thaliana. Plant J. 65, 634-646. doi: 10.1111/j.1365-313X.2010.04449.x

Zhang, C., Xu, Y. Y., Guo, S. Y., Zhu, J. Y., Huan, G., Liu, H. H., et al. (2012). Dynamics of brassinosteroid response modulated by negative regulator LIC in rice. PLOS Genet. 8:e1002686. doi: 10.1371/journal.pgen.100 2686

Zhu, J. Y., Li, Y. Y., Cao, D. M., Yang, H. J., Oh, E. Y., Bi, Y., et al. (2017). The F-box protein KIB1 mediates brassinosteroid-induced inactivation and degradation of 
GSK3-like kinases in Arabidopsis. Mol. Cell 66, 648-657. doi: 10.1016/j.molcel. 2017.05.012

Conflict of Interest Statement: The authors declare that the research was conducted in the absence of any commercial or financial relationships that could be construed as a potential conflict of interest.
Copyright (C) 2017 Xiao, Liu, Zhang, Tong and Chu. This is an open-access article distributed under the terms of the Creative Commons Attribution License (CC BY). The use, distribution or reproduction in other forums is permitted, provided the original author(s) or licensor are credited and that the original publication in this journal is cited, in accordance with accepted academic practice. No use, distribution or reproduction is permitted which does not comply with these terms. 